DOI: https://doi.org/10.46296/ig.v2i3.0007

\title{
EXTRACCIÓN DE ACEITES ESENCIALES DE MANDARINA (CITRUS RETICULATA) Y PALO SANTO (BURSERA GRAVEOLENS) POR EL MÉTODO DE ARRASTRE DE VAPOR
}

\section{EXTRACTION OF MANDARIN (CITRUS RETICULATA) AND PALO SANTO (BURSERA GRAVEOLENS) ESSENTIAL OILS BY THE VAPOR DRAWING METHOD}

\author{
Briones-Sornoza Henry Ricardo ${ }^{1 *}$; Guerrero-Intriago Dumar Andrés² \\ ${ }^{1}$ Universidad Técnica de Manabí, UTM. Portoviejo, Ecuador. \\ ${ }^{2}$ Universidad Técnica de Manabí, UTM. Portoviejo, Ecuador.
}

*Correo: hbriones9653@gmail.com

\begin{abstract}
Resumen
Los aceites esenciales son fracciones líquidas volátiles, generalmente son mezclas homogéneas de hasta 100 compuestos químicos orgánicos, que se obtienen del material vegetal proveniente de los desechos. El presente trabajo tiene como objetivo extraer aceites esenciales de palo santo y de mandarina mediante el método de extracción por arrastre de vapor, para lo cual, se realizó el diseño, construcción y experimentación de un equipo a escala banco, implementando cálculos y fundamentos ingenieriles obtenidos de diferentes fuentes bibliográficas. Las pruebas que se realizaron en el equipo diseñado para la destilación por arrastre de vapor a escala banco presentaron los siguientes resultados: palo santo con rendimientos del 2,7985\% y de $4,3425 \%$, mientras que, la cáscara de mandarina obtuvo un rendimiento de $0,0816 \%$, lo cual indica un resultado muy bajo en comparación al palo santo; sin embargo, comparado con otras investigaciones la extracción de aceite de mandarina obtuvo un mayor rendimiento.
\end{abstract}

Palabras clave: aceite esencial, arrastre de vapor, diseño, extracción, mandarina, palo santo.

\begin{abstract}
Essential oils are volatile liquid fractions, several are homogeneous mixtures of up to 100 organic chemical compounds, which are the products of plant material from waste. The objective of this work is to extract essential oils from palo santo and tangerine using the steam drag extraction method, for which purpose, the design and construction and experimentation of a bank-scale team, implementation of analysis and fundamentals of engineering from different bibliographic sources. The tests that are in the equipment designed for the distillation by steam dragging on a bench scale presented as results yields with the palo santo of $2.7985 \%$ and $4.3425 \%$, however, the tangerine peel obtained a yield of $0.0816 \%$, which indicating a very low result compared to palo santo, however, compared to other bibliographic sources, the extraction of tangerine oil obtained a higher yield.
\end{abstract}

Keywords: essential oil, steam entrainment, design, extraction, tangerine, palo santo.

Información del manuscrito:

Fecha de recepción: 25 de octubre de 2018

Fecha de aceptación: 17 de diciembre de 2018

Fecha de publicación: 10 de enero de 2019 


\section{Introducción}

Los aceites esenciales son fracciones liquidas volátiles, que generalmente son mezclas homogéneas de hasta 100 compuestos químicos orgánicos. Según Navarrete et al., (2010) los aceites esenciales son metabolitos secundarios sintetizados por las plantas, producidos al momento de activarse mecanismos de defensa con respuesta a factores ambientales y ecológicos.

La especie Bursera Graveolens (palo santo), ha sido objeto de estudio por varios investigadores, cuyos resultados obtenidos respecto a la composición de los extractos, del aceite y la actividad farmacológica reportados no son definitivos, mientras que de la naranja y la mandarina se extraen tres aceites esenciales: esencia de naranja 0 mandarina, que se obtiene de la cáscara del fruto; petigrain, que se obtiene de las hojas y ramillas; y esencia de neroli; extraída de las flores (Márquez, 2003).

La protección del medio ambiente se ha convertido en un tema prioritario para los sectores gubernamental e industrial, por ello el presente trabajo se enfoca en el aprovechamiento de los subproductos agroindustriales, siendo estos materiales residuales potenciales de partida para la elaboración de productos comerciales importantes como lo son los aceites esenciales, los cuales pueden brindar rendimientos económicos que contribuirían a minimizar los gastos que supone la gestión de residuos.

\section{Metodología}

\subsection{Procedimiento}

En la figura 1, se muestra el diagrama de bloques para la extracción de aceites esenciales por el método de arrastre de vapor a escala intermedia o banco.

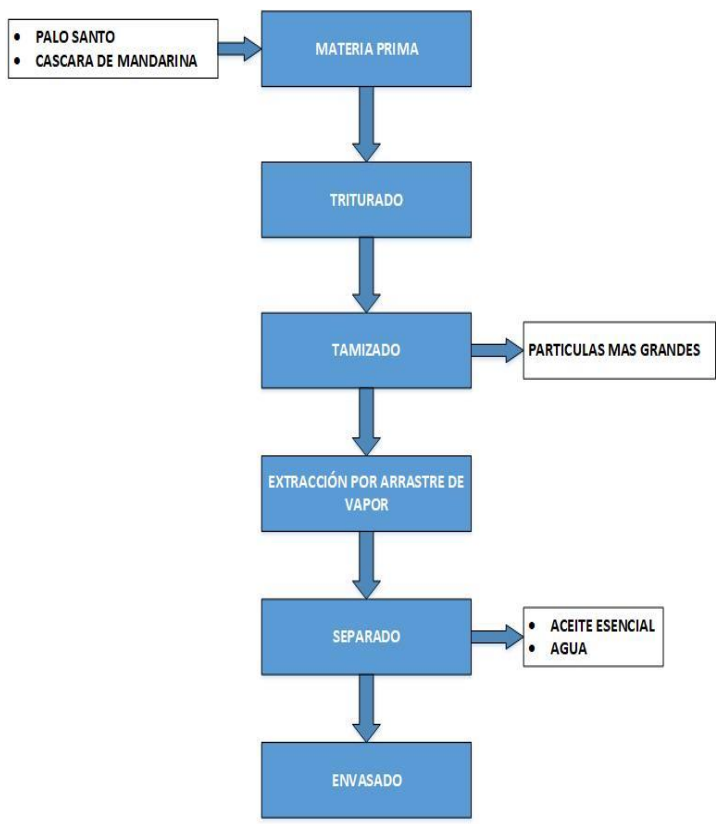

Figura 1. Diagrama de bloques para la extracción de aceites esenciales a escala banco 


\subsubsection{Materia prima}

Para llevar a cabo el proceso de extracción de palo santo y mandarina en el extractor de aceites esenciales por arrastre de vapor a escala banco se realizó la colecta de las muestras de materia prima.

\subsubsection{Triturado}

La madera de palo santo se tritura hasta obtener astillas y virutas que pasaran por el tamizado respectivamente mientras tanto en la mandarina se le extrajo la cáscara para posteriormente secarla y utilizada en el proceso.

\subsubsection{Tamizado}

Se realiza el tamizado de las astillas con el tamiz № 8 y el de las virutas con el tamiz № 4 para obtener las partículas con el tamaño adecuado, logrando así la retención total de las mismas en la parte superior del tanque extractor, el tamizado en la cáscara de mandarina no fue necesario ya que el tamaño de las cáscaras era el adecuado para la rejilla que se encuentra en el equipo.

\subsubsection{Extracción por arrastre de} vapor

Una vez pesadas ambas materias primas se colocan en el equipo para proceder a la extracción por arrastre de vapor, este último proceso tomará un tiempo de 3 horas para una mayor eficiencia.

\subsubsection{Separado}

El método de separación empleado utiliza un embudo de decantación, el cual se deja reposando por un lapso de 1 hora, obtenidas las dos fases (aceite y agua) se procede a separar el agua del aceite lentamente.

\subsubsection{Envasado}

El aceite obtenido será envasado en frascos de color ámbar para preservar la esencia, y la composición.

\subsection{Diseño del extractor por arrastre de vapor}

Antes de realizar el diseño del extractor hay que tener en cuenta la tabla 1, correspondiente al uso de simbología y sus respectivos significados y unidades. 
Tabla 1.

Simbología del proceso

\begin{tabular}{|c|c|c|}
\hline Simbología & Significado & Unidades \\
\hline Ve & Volumen del recipiente & $\mathrm{m}^{3}$ \\
\hline$r$ & Radio del recipiente & $\mathrm{m}$ \\
\hline h & Altura del recipiente & $\mathrm{m}$ \\
\hline Tvi & Temperatura vapor inicial & ${ }^{\circ} \mathrm{C}$ \\
\hline Tac & Temperatura agua inicial & ${ }^{\circ} \mathrm{C}$ \\
\hline Trc & Temperatura vapor condensado & ${ }^{\circ} \mathrm{C}$ \\
\hline Taf & Temperatura agua fría & ${ }^{\circ} \mathrm{C}$ \\
\hline LN & Logaritmo neperiano & Asimétrica \\
\hline $\mathrm{mv}$ & Masa de vapor requerida para la extracción & $\mathrm{kg} / \mathrm{s}$ \\
\hline Dv & Demanda de vapor densidad el agua & $\mathrm{m}^{3} / \mathrm{s}$ \\
\hline Pagua & Densidad el agua & $\mathrm{kg} / \mathrm{m}^{3}$ \\
\hline Cmat & Masa de la materia prima & $\mathrm{Kg}$ \\
\hline $\mathrm{Fa}$ & Factor de aproximación & $2.5 \mathrm{E}-7 \mathrm{~m}^{3} / \mathrm{s}^{*} \mathrm{~kg}$ \\
\hline$\dot{m a}$ & Masa del agua & $\mathrm{kg} / \mathrm{s}$ \\
\hline$\dot{\mathrm{m}}$ & Masa del material a calentar & $\mathrm{Kg} / \mathrm{s}$ \\
\hline$\lambda$ & Variación de entalpias & $(\hat{\mathrm{H}} 110-\hat{\mathrm{H}} 30) \mathrm{kJ} / \mathrm{kg}$ \\
\hline cp & Calor especifico de agua & $4.184 \mathrm{~kJ} / \mathrm{kg}^{\circ} \mathrm{C}$ \\
\hline$\Delta \mathbf{T}$ & Variación de temperatura & ${ }^{\circ} \mathrm{C}$ \\
\hline$Q \mathbf{r}$ & Calor requerido & $\mathrm{kJ} / \mathrm{s}$ \\
\hline $\mathrm{AM}$ & Área media & $\mathrm{m}$ \\
\hline$\Delta \mathrm{TM}$ & Variación de temperatura media & ${ }^{\circ} \mathrm{C}$ \\
\hline$U$ & Calor latente de vaporización & $\mathrm{kJ} / \mathrm{kg}$ \\
\hline D & Diámetro del intercambiador de calor & $\mathrm{cm}$ \\
\hline Qc & $\begin{array}{l}\text { Calor requerido para condensar a } \\
\text { temperatura de } 25 \text { a } 100^{\circ} \mathrm{C}\end{array}$ & $\mathrm{J}$ \\
\hline Qe & $\begin{array}{l}\text { Calor requerido para mantener la } \\
\text { condensación }\end{array}$ & $\mathrm{J}$ \\
\hline QT & Calor total & $\mathrm{J}$ \\
\hline Qp & Calor que pierde & $\mathrm{J}$ \\
\hline$\varepsilon$ & Eficiencia del intercambiador de calor & $\%$ \\
\hline Tce & Temperatura caliente de entrada & ${ }^{\circ} \mathrm{C}$ \\
\hline Tcs & Temperatura caliente de salida & ${ }^{\circ} \mathrm{C}$ \\
\hline$T f e$ & Temperatura fría de entrada & ${ }^{\circ} \mathrm{C}$ \\
\hline Tfs & Temperatura fría de salida & ${ }^{\circ} \mathrm{C}$ \\
\hline
\end{tabular}

Para el diseño del equipo extractor

ecuaciones de diseño planteadas en se realizaron cálculos a partir de las

la tabla 2.

Tabla 2.

Ecuaciones para el diseño del extractor por arrastre de vapor.

\begin{tabular}{|l|c|}
\hline \multicolumn{2}{|c|}{ Ecuaciones de diseño } \\
\hline $\begin{array}{l}\text { Variación de temperatura media } \\
\text { logaritmica del intercambiador de } \\
\text { calor }\end{array}$ & $\Delta T M=\frac{(\mathrm{Tvi}-\mathrm{Tac})-(\mathrm{Tvc}-\mathrm{Taf})}{\frac{\mathrm{LN}(\mathrm{Tvi}-\mathrm{Tac})}{\mathrm{Tvc}-\mathrm{Taf}}}$ \\
\hline Masa del agua & $\mathrm{ma}=\frac{\mathrm{m} * \lambda}{\Delta T * c p}$ \\
\hline Calor requerido & $\mathrm{Qr}=\mathrm{m} * \mathrm{cp} * \Lambda \mathrm{T}$ \\
\hline Área Media & $\Lambda \mathrm{M}=\frac{\mathrm{Qr}}{\mathrm{U} * \Delta \mathrm{TM}}$ \\
\hline Diámetro & $D=\sqrt{\frac{4(\Lambda \mathrm{M})}{\pi}}$ \\
\hline Volumen del vapor requerido & $\mathrm{Dv}=\mathrm{Cmat} * \mathrm{Fa}$ \\
\hline Masa del vapor requerido & $\mathrm{mv}=\mathrm{Dv} * \rho \mathrm{H} 20$ \\
\hline Volumen de recipientes cilindricos & $V e=\pi r^{2} h$ \\
\hline
\end{tabular}




\subsection{Cálculos de balances}

En la tabla 3 se pueden observar los cálculos para los respectivos balances de masa y de energía.

\section{Tabla 3.}

Ecuaciones de balance de masa y energía

\begin{tabular}{|l|c|}
\hline \multicolumn{2}{|c|}{ Balance de masa } \\
\hline $\begin{array}{l}\text { Ecuación de igualación de } \\
\text { masa del sistema }\end{array}$ & $\begin{array}{l}\text { Masa entrada }=\text { Masa salida + } \\
\text { Masa acumulada }\end{array}$ \\
\hline \multicolumn{2}{|c|}{ Balance de energia en el generador de vapor } \\
\hline Calentamiento & $\mathrm{Qc}=\mathrm{m} * \mathrm{cp} * \Delta \mathrm{T}$ \\
\hline Evaporación & $\mathrm{Qe}=\mathrm{m} \cdot \lambda$ \\
\hline $\begin{array}{l}\text { calor total consumido en el } \\
\text { generador de vapor }\end{array}$ & $\mathrm{QT}=\mathrm{Qc}+\mathrm{Qe}$ \\
\hline
\end{tabular}

\subsection{Rendimientos de la utilizada (g).}

\section{experimentación}

La siguiente ecuación hace referencia a la cantidad de producto obtenido a partir de una determinada cantidad de materia prima que ha sido sometida al proceso de extracción por arrastre de vapor.

$$
\% \text { Rendimiento }=\frac{a e \times \rho_{a e}}{\mathrm{mmpu}} \times 100
$$

Donde:

ae: aceite esencial extraído $(\mathrm{mL})$. $\rho_{a e}:$ Densidad del aceite esencial $(\mathrm{g} / \mathrm{mL})$.

mmpu: Masa de materia prima

\section{Resultados y discusión}

\subsection{Diseño del equipo de arrastre} de vapor

Tabla 4.

Características del diseño del extractor

\begin{tabular}{|c|c|}
\hline \multicolumn{2}{|c|}{ Ecuaciones de diseño } \\
\hline $\begin{array}{l}\text { Variación de temperatura media } \\
\text { logarítmica del intercambiador } \\
\text { de calor }\end{array}$ & $\Delta T M=19.54^{\circ} \mathrm{C}$ \\
\hline Masa del agua & tha $=2.676737 \times 10^{-4} \mathrm{Kg} / \mathrm{s}$ \\
\hline Calor requerido & $\mathrm{Qr}=0.03919 \mathrm{KJ} / \mathrm{s}$ \\
\hline Área Media & $\Lambda \mathrm{M}=1.57336910^{-3} \mathrm{~m}^{2}$ \\
\hline Diámetro & $D=0.04475 m$ \\
\hline Volumen del vapor requerido & $D v=1.25 \times 10^{-4} \mathrm{~m}^{3} / \mathrm{s}$ \\
\hline Masa del vapor requerido & $\mathrm{mv}=1.25 \times 10^{-4} \mathrm{~kg} / \mathrm{s}$ \\
\hline $\begin{array}{l}\text { Volumen de recipientes } \\
\text { cilindricos } \\
\text { a) Volumen del extractor } \\
\text { b) Volumen del extractor }\end{array}$ & $\begin{array}{l}\text { a) } V e=0.01039 \mathrm{~m}^{2} \\
\text { b) } V e=0.000318086 \mathrm{~m}^{3}\end{array}$ \\
\hline
\end{tabular}




\subsubsection{Cálculo de la masa}

En el cálculo de la masa de los aceites esenciales se utilizó el volumen máximo extraído en las especies vegetales y las densidades fueron tomadas bibliográficamente de trabajos previamente realizados.

La densidad del aceite esencial de palo santo fue un dato bibliográfico que presentó un valor de $0,965 \mathrm{~g} / \mathrm{mL}$ (Dellacassa, 2010).

La densidad del aceite esencial de mandarina fue un dato bibliográfico que presentó un valor de $0,816 \mathrm{~g} / \mathrm{mL}$ (Fouad \& da Camara, 2017; González, 2004).

\subsubsection{Masa del aceite esencial} de palo santo

$$
\begin{gathered}
m=\rho_{\mathrm{ae}} * V \\
m=0,965 \mathrm{~g} / \mathrm{mL} * 4,5 \mathrm{~mL} \\
m=4,3425 \mathrm{~g}
\end{gathered}
$$

\subsubsection{Masa del aceite esencial} de cáscara de mandarina

$$
\begin{gathered}
m=\rho_{\mathrm{ae}} * V \\
m=0,816 \mathrm{~g} / \mathrm{mL} * 0,5 \mathrm{~mL} \\
m=0,408 \mathrm{~g}
\end{gathered}
$$

Dónde:

$\rho_{\text {ae: }}$ Densidad del aceite esencial $(\mathrm{g} / \mathrm{mL})$.
V: Volumen del aceite esencial $(\mathrm{mL})$.

m: masa del aceite (g).

\subsection{Dimensionamiento del} extractor de aceite esencial

En la tabla 5 se encuentra el dimensionamiento del equipo extractor de aceites esenciales a escala banco realizado en el presente trabajo.

\section{Tabla 5.}

Dimensiones del equipo extractor

\begin{tabular}{|l|c|c|c|}
\hline Material de construcción & Acero inoxidable AISI 304 \\
\hline \multicolumn{4}{|c|}{ Dimensiones } \\
\hline Elementos dimensiones & $\begin{array}{c}\text { Diámetro } \\
(\mathrm{m})\end{array}$ & $\begin{array}{c}\text { Altura } \\
(\mathrm{m})\end{array}$ & $\begin{array}{c}\text { Volumen } \\
\left(\mathrm{m}^{3}\right)\end{array}$ \\
\hline Cámara extractora & 0.21 & 0.30 & 0.01039 \\
\hline Intercambiador de calor & 0.045 & 0.20 & 0.000318 \\
\hline
\end{tabular}

\subsection{Cálculos de balances}

\section{Tabla 6.}

Resultados del balance de masa y de energía

\begin{tabular}{|l|c|}
\hline \multicolumn{2}{|c|}{ Balance de masa } \\
\hline $\begin{array}{l}\text { Ecuación de igualación de } \\
\text { masa del sistema }\end{array}$ & $3500 \mathrm{~g}=1275 \mathrm{~g}+2225 \mathrm{~g}$ \\
\hline \multicolumn{2}{|c|}{ Balance de energía en el generador de vapor } \\
\hline Calentamiento & $\mathrm{Qc}=1098300 \mathrm{~J}$ \\
\hline Evaporación & $\mathrm{Qe}=2876910 \mathrm{~J}$ \\
\hline $\begin{array}{l}\text { calor total consumido en el } \\
\text { generador de vapor }\end{array}$ & $\mathrm{QT}=3975210 \mathrm{~J}$ \\
\hline
\end{tabular}

\subsection{Rendimientos}

\subsubsection{Rendimiento del palo santo}

De los datos experimentales se obtuvo que cuando se trabaja con astillas (500gr) después de 3 horas de extracción, la cantidad de aceite esencial obtenido fue de $14,5 \mathrm{~mL}$, 
por lo tanto, el rendimiento se calculó de la siguiente forma:

$$
\begin{gathered}
\% R=\frac{14,5 \mathrm{ml} \times 0.965 \mathrm{~g} / \mathrm{mL}}{500 \mathrm{~g}} \times 100= \\
2,7985 \%
\end{gathered}
$$

Cuando se trabajó con madera en forma de virutas (100gr) se obtuvieron $4,5 \mathrm{~mL}$ de aceite esencial recuperado, por lo tanto, el rendimiento del proceso se calculó así:

$$
\begin{gathered}
\% R=\frac{4.5 \mathrm{ml} \times 0.965 \mathrm{~g} / \mathrm{mL}}{100 \mathrm{~g}} \times 100= \\
4,3425 \%
\end{gathered}
$$

\subsubsection{Rendimiento de la cáscara de mandarina}

En los datos experimentales se obtuvo $0,5 \mathrm{~mL}$ de aceite esencial después de una extracción de 3 horas, el rendimiento se lo determinó de la siguiente forma:

$$
\begin{gathered}
\% R=\frac{0.5 \mathrm{ml} \times 0.816 \mathrm{~g} / \mathrm{mL}}{500 \mathrm{~g}} \times 100= \\
0,0816 \%
\end{gathered}
$$

\subsubsection{Rendimiento del equipo extractor}

Refleja que el desempeño del equipo extractor en cuanto al aprovechamiento del calor suministrado al sistema.

$$
\varepsilon=1-\frac{\mathrm{Qp}}{\mathrm{QT}} \times 100
$$

Para el sistema se considera una pérdida del $10 \%$, por lo tanto:

$$
\begin{gathered}
Q p=0,1 Q \top \\
Q p=0,1(3975210 \mathrm{~J}) \\
Q p=397521 \mathrm{~J} \\
\varepsilon=1-\frac{397521 \mathrm{~J}}{3975210 \mathrm{~J}} \times 100 \\
\varepsilon=90 \%
\end{gathered}
$$

\subsubsection{Rendimiento}

del

\section{intercambiador de calor}

$$
\begin{gathered}
\varepsilon=\frac{\text { Tce }- \text { Tcs }}{\text { Tce }- \text { Tfe }} \times 100 \% \\
\varepsilon=\frac{110-35}{110-20} \times 100 \% \\
\varepsilon=83,33 \%
\end{gathered}
$$

\subsection{Resultados obtenidos de las extracciones}

El proceso de obtención de aceites a partir de palo santo se realizó tanto en astillas como en virutas según lo expuesto previamente; se pueden observar los rendimientos obtenidos a partir de astillas realizando 3 extracciones para las diferentes cantidades de materia prima a procesar. 
Tabla 7.

Extracción de aceite palo santo en astillas durante 1 hora y $3500 \mathrm{~mL}$ de agua

\begin{tabular}{|c|c|c|c|c|c|c|}
\hline \multicolumn{7}{|c|}{ PALO SANTO } \\
\hline $\begin{array}{c}\text { Materia } \\
\text { prima } \\
(\mathrm{gr})\end{array}$ & $\begin{array}{c}\text { Destilado } \\
(\mathrm{ml})\end{array}$ & $\begin{array}{c}\text { Aceite } \\
\text { recuperado } \\
(\mathrm{ml})\end{array}$ & $\begin{array}{c}\text { Aceite } \\
\text { promedio } \\
\text { recuperado } \\
(\mathrm{ml})\end{array}$ & $\begin{array}{c}\text { Rendimiento } \\
(\%)\end{array}$ \\
\hline 300 & 500 & 1.5 & 1.8 & 1.6 & $\mathbf{1 . 6 3}$ & $\mathbf{0 . 5 2 4 3}$ \\
\hline 400 & 500 & 3.7 & 3.5 & 3.6 & 3.6 & $\mathbf{0 . 8 6 8 5}$ \\
\hline 500 & 500 & 4.7 & 5.3 & 4.9 & $\mathbf{4 . 9 7}$ & $\mathbf{0 . 9 5 9 2}$ \\
\hline
\end{tabular}

En la producción de aceite de mandarina se utilizó la cascara seca, donde se realizaron 3 repeticiones a diferentes cantidades de materia prima observadas en la tabla 8, donde el tiempo de extracción fue de 3 horas.

Tabla 8.

Extracción de aceite de mandarina durante 3 horas y $3500 \mathrm{~mL}$ de agua

\begin{tabular}{|c|c|c|c|c|c|c|}
\hline \multicolumn{7}{|c|}{ Mandarina } \\
\hline $\begin{array}{c}\text { Materia } \\
\text { prima } \\
(\mathrm{gr})\end{array}$ & $\begin{array}{c}\text { Destilado } \\
(\mathrm{ml})\end{array}$ & \multicolumn{2}{|c|}{$\begin{array}{c}\text { Aceite } \\
\text { recuperado } \\
(\mathrm{ml})\end{array}$} & $\begin{array}{c}\text { Aceite } \\
\text { promedio } \\
\text { recuperado } \\
(\mathrm{ml})\end{array}$ & $\begin{array}{c}\text { Rendimiento } \\
(\%)\end{array}$ \\
\hline 300 & 1500 & 0.1 & 0.3 & 0.2 & 0.2 & 0.0544 \\
\hline 400 & 1350 & 0.2 & 0.4 & 0.3 & 0.3 & 0.0612 \\
\hline 500 & 1250 & 0.4 & 0.6 & 0.5 & 0.5 & 0.0816 \\
\hline
\end{tabular}

En la tabla 9 se observan los resultados obtenidos durante las extracciones realizadas para el palo santo en forma de virutas con 3 repeticiones para cada cantidad de materia prima durante el tiempo de 3 horas de extracción.
Tabla 9.

Extracción de aceite de palo santo en tiempo de 3 horas y $3500 \mathrm{~mL}$ de agua

\begin{tabular}{|c|c|c|c|c|c|c|}
\hline \multicolumn{7}{|c|}{ PALO SANTO } \\
\hline $\begin{array}{c}\text { Materia } \\
\text { prima } \\
(\mathrm{gr})\end{array}$ & $\begin{array}{c}\text { Destilado } \\
(\mathrm{ml})\end{array}$ & \multicolumn{2}{|c|}{$\begin{array}{c}\text { Aceite } \\
\text { recuperado } \\
(\mathrm{ml})\end{array}$} & $\begin{array}{c}\text { Aceite } \\
\text { promedio } \\
\text { recuperado } \\
(\mathrm{ml})\end{array}$ & $\begin{array}{c}\text { Rendimiento } \\
(\%)\end{array}$ \\
\hline 500 & 1500 & $\begin{array}{r}13 . \\
8\end{array}$ & 14 & $\begin{array}{c}14 . \\
2\end{array}$ & 14 & 2.7985 \\
\hline 300 & 1350 & 9.5 & 9.3 & 9.2 & 9.33 & 3.001 \\
\hline 100 & 1250 & 4.6 & 4.2 & 4.7 & 4.5 & 4.3425 \\
\hline
\end{tabular}

Las pruebas que se realizaron en el equipo diseñado para la destilación por arrastre de vapor a escala banco presentaron diferentes rendimientos para las dos materias primas empleadas. Con el aceite esencial de palo santo se obtuvo un rendimiento del $2,7985 \%$ para las astillas y $4,3425 \%$ para las virutas, comprobándose que la utilización de las virutas logra una mayor obtención de aceite esencial. Por lo tanto, en comparación a los datos expuestos por Mora (2014), de $2,77 \%$ en astillas y $3,49 \%$ en virutas, los rendimientos efectuados en el presente proyecto fueron superiores. Al trabajar con la cáscara de mandarina el rendimiento fue de $0,0816 \%$, lo cual nos indica un resultado muy bajo en comparación con el rendimiento obtenido para el palo santo, esto es debido a que se trata de una materia prima de origen cítrico y además no posee 
Revista Científica "INGENIAR": Ingeniería, Tecnología e Investigación. Vol 2, Num 3 (ene-jun) ISSN: 2737-6249 Extracción de aceites esenciales de mandarina (Citrus reticulata) y palo santo (Bursera graveolens) por el

cantidades considerables de aceites esenciales (Navarrete et al., 2010).

Finalmente, con los datos expuestos se demuestra que la instalación del equipo extractor de aceites esenciales por arrastre de vapor para especies vegetales a escala banco tiene vialidad satisfactoria para la producción de aceites, ya que se obtienen resultados positivos superando a los datos bibliográficos expuestos anteriormente.

\section{Conclusiones}

Los cálculos para el diseño del proceso se realizaron considerando la cantidad de materia prima a procesar y la cantidad de vapor que se va a condensar. En función de la cantidad de vapor que se genera se procedió a calcular el área del intercambiador de calor para que ocurra el proceso de condensación.

Las condiciones óptimas de operación para la extracción de aceites esenciales de especies vegetales (palo santo y mandarina) están relacionadas con respecto al tiempo y a la cantidad de materia prima que se introducirá en el tanque extractor, cabe considerar que la materia prima que contenga baja cantidad de aceites esenciales producirá un rendimiento no favorable.

Se evaluó el rendimiento para cada una de las materias primas donde se realizaron extracciones por triplicado en diferentes concentraciones, estableciendo que el rendimiento obtenido en la extracción del aceite esencial de palo santo (Bursera graveolens) es mayor con respecto al de mandarina (Citrus reticulata).

\section{Bibliografía}

Dellacassa,

E.

(2010).

Normalización de productos naturales obtenidos de especies de la flora aromática latinoamericana: proyecto CYTED IV. 20. EdiPUCRS.

Fouad, H. A., \& da Camara, C. A. (2017). Chemical composition and bioactivity of peel oils from Citrus aurantiifolia and Citrus reticulata and enantiomers of their major constituent against Sitophilus zeamais (Coleoptera: Curculionidae). Journal of Stored Products Research, 73, 30-36.

González-Villa, Á. (2004). Obtención de aceites esenciales $y$ extractos etanólicos de plantas del Amazonas. Ingeniería Química.

Márquez, L. (2003). Extracción del aceite esencial de mandarina (Citrus reticulata) utilizando 
dióxido de carbono en condición supercrítica como solvente. Trabajo especial de Grado, Universidad Central de Venezuela, CaracasVenezuela.

Mora-Moscoso, G. A. (2014). Diseño de una planta para la extracción del aceite esencial de palo santo (Bursera graveolens) mediante destilación por arrastre de vapor (Bachelor's thesis, Escuela Politécnica Nacional, Quito, Ecuador).
Navarrete, C., Gil, J., Durango, D., \& Garcia, C. (2010). Extracción y caracterización del aceite esencial de mandarina obtenido de residuos agroindustriales. Dyna, 77(162), 85-92. 